\title{
On the Scaling Behavior of the Abelian Sandpile Model
}

\author{
Barbara Drossel \\ Department of Physics, University of Manchester, Manchester M13 9PL, England
}

\begin{abstract}
The abelian sandpile model in two dimensions does not show the type of critical behavior familar from equilibrium systems. Rather, the properties of the stationary state follow from the condition that an avalanche started at a distance $r$ from the system boundary has a probability proportional to $1 / \sqrt{r}$ to reach the boundary. As a consequence, the scaling behavior of the model can be obtained from evaluating dissipative avalanches alone, allowing not only to determine the values of all exponents, but showing also the breakdown of finite-size scaling.

PACS numbers: 64.60.Lx
\end{abstract}

Since its introduction in 1987, the sandpile model has been considered as the prototype of a self-organized critical (SOC) system [1]. Computer simulations suggest that irrespective of the initial conditions and of details of the model rules, the system self organizes into a "critical" state with a power-law size distribution of avalanches. The concept of SOC is thought to explain the frequent occurrence of power laws in nature.

Considerable effort has been made to determine the scaling behavior of the two-dimensional abelian sandpile model. The advantage of this model is that several of its properties can be calculated analytically 2,3]. However, the exponent $\tau_{r}$ characterizing the distribution of avalanche radii, and related exponents for the distribution of the avalanche duration $t$ and the number of topplings $s$ during an avalanche have resisted any attempt of an analytical calculation. The numerical determination of these exponents is hampered by the fact that the double logarithmic plots show slopes that increase with increasing system size, until finite-size effects set in, indicating that the asymptotic scaling behavior does not yet occur for the system sizes accessible to computer simulations. Thus, the predictions for the value of $\tau_{s}$ vary between 1.22 [4] and 1.27 [5] or even 1.29 [6]. The latter two results where obtained under the assumption that the system displays finite-size scaling (FSS).

Recently, evidence was found that the abelian sandpile model does not display FSS [7]. This result was obtained from an investigation of multifractal spectra. Indeed, there is no a priori reason why the abelian sandpile model should display FSS. The concept of FSS has its origin in equilibrium critical phenomena where a small finite system cannot be distinguished from a small part of a large system. However, this is not the case for the abelian sandpile model in two dimensions. In a small system, the sites that participate in an avalanche may topple a few times during the duration of the avalanche. In a larger system, the number of topplings per site during a large avalanche is larger. Thus, locally collected data (i.e. the number of topplings of a given site) contains information about the size of the system. Also, finite-size scaling is based on the assumption that boundaries play no special role in the system. However, the boundaries of the abelian sandpile model play an essential role as they are the only place where sand can leave the system.

It is the purpose of this letter to elucidate the scaling behavior of the abelian sandpile model. Since boundaries play a special role in the system, it is essential to consider avalanches that reach the boundaries of the system separately from those that don't. It turns out that the dissipative avalanches display beautiful power laws. These power laws imply that each avalanche started at a distance $r$ from the system boundary has a probability proportional to $1 / \sqrt{r}$ to reach the boundary and, if it does so, dissipates on an average $\sqrt{r}$ sand grains. From the scaling behavior of dissipative avalanches, we obtain the values of the critical exponents characterizing the system, and we see compelling evidence for the violation of FSS.

The two-dimensional abelian sandpile model is defined on a square lattice with $L^{2}$ sites. At each site $i$ an integer variable $z_{i}$ represents the number of grains. Grains are added individually to randomly chosen sites of the system. When the number of grains at a site $i$ exceeds $z_{c}=3$, site $i$ is unstable and topples, its height being reduced to $z_{i}-4$, and the heights $\left\{z_{j}\right\}$ of all four nearest neighbors being increased by one. If $i$ is a boundary site with $l<4$ neighbors, $4-l$ grains leave the system. If a neighbor $j$ becomes unstable due to the addition of a grain, it also topples, and the avalanche stops when a new stable configuration is reached. During an avalanche, no new grains are added to the system. The size $s$ of an avalanche is defined to be the total number of topplings. The radius of an avalanche is in this paper taken to be the maximum distance of toppled sites from the starting site of the avalanche. It has proven useful to decompose an avalanche into "waves of toppling" [9]. The $n$th wave of toppling begins when the starting point of an avalanche topples for the $n$th time, and all those sites belong to it that topple immediately after a nearest neighbor that belongs to the same wave has toppled.

Let us first discuss the radius distribution of avalanches, $n_{r}(r, L)$. Since its asymptotic (i.e., $L \rightarrow \infty$ ) behavior is difficult to extract from simulation data, recent results for the exponent $\tau_{r}$ vary between $7 / 5$ [7] and $5 / 3$ [6]. In [8], a version of the abelian sandpile model was studied where sand grains were only dropped at the center of the system. It was found that the frac- 
tion $1 / \sqrt{L}$ of all avalanches reach the boundaries. This finding would imply that $\tau_{r}=3 / 2$, just as predicted in [9]. This is indeed the correct exponent, as a separate evaluation of dissipative and nondissipative avalanches shows. We denote by $n_{r}^{(1)}(r, L)$ the radius distribution of nondissipative avalanches, i.e., those avalanches that do not reach the boundaries. The distribution of dissipative avalanches is denoted $n_{r}^{(2)}(r, L)$. Normalization of the radius distribution requires

$$
\int_{1}^{\infty}\left[n_{r}^{(1)}(r, L)+n_{r}^{(2)}(r, L)\right] d r=1 .
$$

Fig. 1 shows the result of computer simulations for $n_{r}^{(2)}(r, L)$.

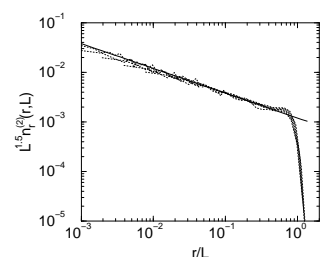

FIG. 1. The radius distribution of avalanches that touch the boundaries of the system, for $L=128,256,512,1024$, 2048. The thick solid line is a power law with an exponent $-1 / 2$.

The distribution $n_{r}^{(2)}(r, L)$ has the form

$$
n_{r}^{(2)}(r, L) \sim L^{-3 / 2} g(r / L) .
$$

The prefactor is such that the total weight of $n_{r}^{(2)}(r, L)$ is $\int_{1}^{L} n_{r}^{(2)}(r, L) d r \sim 1 / \sqrt{L}$, in agreement with the result in 8 that the fraction $1 / \sqrt{L}$ of all avalanches reach the boundaries. Since grains are dropped randomly into the system, the probability that an avalanche is triggered at a fixed distance $r \ll L$ from the boundary is proportional to $1 / L$, leading to $n_{r}^{(2)}(r, L)=(1 / L) f(r)$ for $r \ll L$. Together with Eq. (11), this gives $g(r / L) \propto(r / L)^{-1 / 2}$ for small $r / L$. From Fig. 1, it can be seen that this $1 / \sqrt{r}$ behavior extends almost over the entire range of $r$ values. An avalanche triggered at a distance $r$ from the boundary consequently has a probability proportional to $1 / \sqrt{r}$ to reach the boundary, and if it reaches the boundary it dissipates on an average of the order $\sqrt{r}$ sand grains.

Fig. 2 shows the radius distribution of avalanches that do not reach the boundaries. For the system sizes studied in the simulations, no power law is visible. The slope seems to become steeper with increasing $r$. (The last part of each curve, which begins at the point where it splits from the curves for larger system sizes, is due to finite-size effects and it must be ignored in the subsequent discussion which refers to the thermodynamic limit $L \rightarrow \infty$.) However, the curves $n_{r}^{(1)}(r, L)$ must approach a power law $n_{r}^{(1)}(r, \infty) \sim r^{-\tau_{r}}$ for sufficiently large $r$. The reason is that the last wave of topplings of an avalanche has been proved to be distributed according to a power law $r^{-7 / 4}$ [10], and $n_{r}^{(1)}(r, \infty)$ cannot be steeper than this. From the above results for $n_{r}^{(2)}(r, L)$, we can even deduce the value of $\tau_{r}$ : The probability that an avalanche triggered at distance $r$ from the boundary reaches the boundary is proportional to $L n_{r}^{(2)}(r, L)$, and it is identical to the probability that an avalanche triggered in the interior of the system reaches at least a radius $r$, which is given by $\int_{r}^{\infty} n_{r}^{(1)}\left(r^{\prime}, \infty\right) d r^{\prime}$. The reason is that the landscape of height values in the abelian sandpile model does not show long-range correlations. Rather, correlations decay as fast as $r^{-4}$ [2], implying that avalanches spread in the same way everywhere in the system, as long as they do not encounter the boundaries. Consequently, $n_{r}^{(1)}(r, \infty)$ must fall off as $r^{-3 / 2}$ for sufficiently large $r$, fixing the value of $\tau_{r}=3 / 2$. Fig. 2 2 shows that this asymptotic value is barely reached for the system sizes accessible to computer simulations. This explains why evaluations based on the complete radius distribution $n_{r}^{(1)}(r, L)+n_{r}^{(2)}(r, L)$ do not reveal the correct exponent.

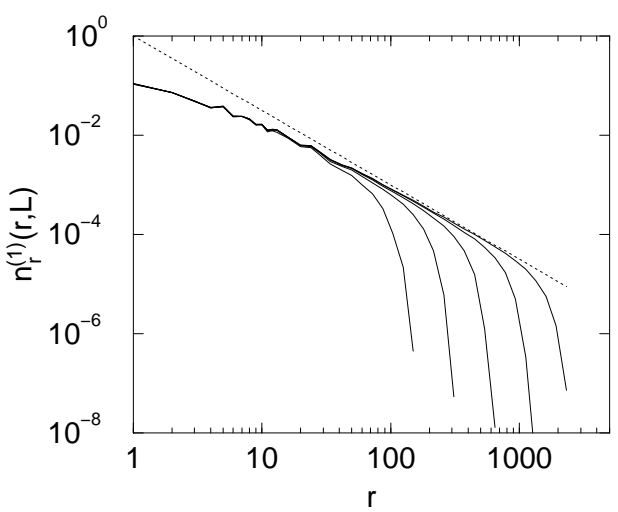

FIG. 2. The radius distribution of avalanches that do not touch the boundaries of the system, for $L=128,256,512$, 1024, 2048 from left to right. The dotted line is a power law with the exponent $-3 / 2$.

The distribution of the number of topplings $n_{s}(s, L)=$ $n_{s}^{(1)}(s, L)+n_{s}^{(2)}(s, L)$ in avalanches can be analyzed in a similar way. The superscript (1) refers again to nondissipative, the superscript (2) to dissipative avalanches. If the number of topplings $s$ in an avalanche of radius $r$ was always of the order $r^{D}$, simple scaling would hold, resulting in a FSS form $n_{s}(s, L) \sim s^{-\tau_{s}} \mathcal{C}\left(s / L^{D}\right)$ and a scaling relation $D=\left(\tau_{r}-1\right) /\left(\tau_{s}-1\right)$. The breakdown of simple scaling was pointed out for the first time in 8,7]. It can best be visualized by looking at the distribution of the number of topplings $n_{s}^{\text {(spanning) }}(s, L)$ of those avalanches that span the entire system, i.e. that touch all four edges of the boundary. These avalanches have a radius of the order $L$. 


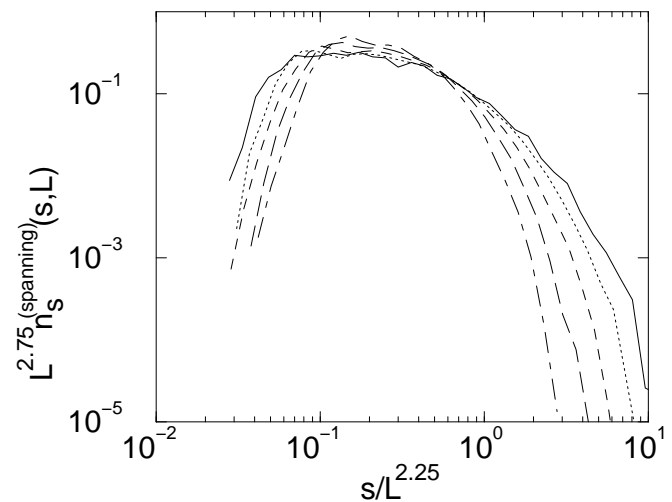

FIG. 3. The distribution $n_{s}^{\text {(spanning) }}(s, L)$ of the number of topplings in system-spanning avalanches for $L=$ $2048,1024,512,256,128$ (from widest to narrowest curve).

Evaluation of the curves represented in Fig. 3 shows that the maximum scales as $L^{D}$ with $D=9 / 4$, the lower cutoff as $L^{2}$, and the upper cutoff as $L^{11 / 4}$. These exponents are obtained by scaling $s$ in such a way that the maxima, the left tails, or the right tails collapse. The mean scales roughly as $L^{5 / 2}$. Clearly, a complete scaling collapse would only be possible if the four quantities were characterized by the same exponent. The scaling behavior of the mean implies

$$
\bar{s}=\int_{1}^{\infty} s n_{s}(s, L) d s \sim L^{2},
$$

in agreement with the analytical result given in [2].

Fig. 6 shows the distributions $n_{s}^{(2)}(s, L)$ of the number of topplings in dissipative avalanches.

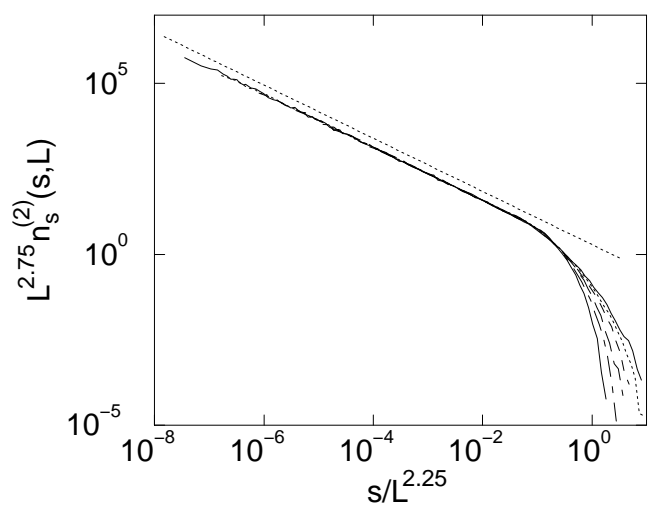

FIG. 4. The distribution $n_{s}^{(2)}(s, L)$ of the number of topplings in dissipative avalanches for $L=$ $2048,1024,512,256,128,64$ (from widest to narrowest curve). The dotted line is a power law with the exponent $-7 / 9$.

The curves have the form $n_{s}^{(2)}(s) \sim L^{-1} s^{-7 / 9}$ for sufficiently small $s$, and then have a cutoff that does not display FSS but is similar to the one for spanning avalanches. The total weight of dissipative avalanches, $1 / \sqrt{L}$, is proportional to $\int_{1}^{L^{9 / 4}} n_{s}^{(2)}(s, L) d s$. The expo- nent $\tau_{s}$ can be derived from the condition

$$
s^{1-1 / D} L n_{s}^{(2)}(s, L) \sim \int_{s}^{\infty} n_{s}^{(1)}\left(s^{\prime}, \infty\right) d s^{\prime},
$$

giving $\tau_{s}=11 / 9$, which agrees very well with the value obtained by Manna [4]. The asymptotic slope $-\tau_{s}$ is barely visible in $n_{s}^{(1)}(s, L)$ for the system sizes accessible to computer simulations. For the value of the exponent $\tau_{s}$, the multiscaling features are irrelevant, since only the exponent $D=9 / 4$ enters the evaluation of $\tau_{s}$. However, the multiscaling behavior is relevant for the result $\bar{s}=L^{2}$, which stands in no relation to the value of $\tau_{s}$.

The failure of FSS in the system is due to a broad distribution of the number of waves of toppling in avalanches. Single waves of toppling display FSS, as shown in Fig. 5 for the first wave of toppling.

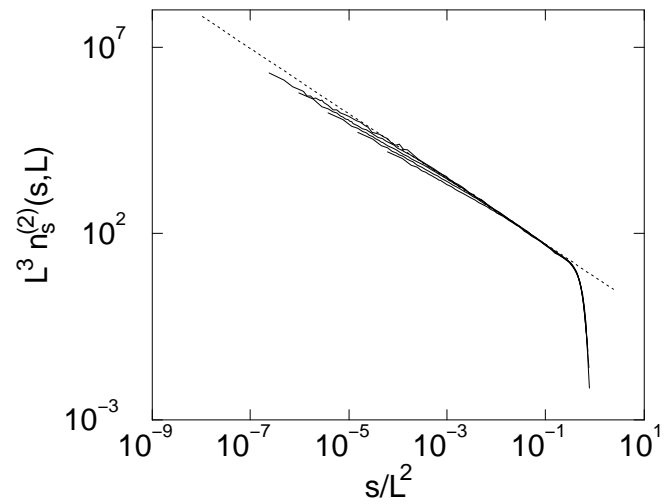

FIG. 5. The distribution $n_{s}^{(2)}(s, L)$ of the number of topplings in dissipative first waves for $L=2048,1024,512,256,128$. The dotted line is a power law with the exponent $-7 / 8$.

The scaling with $L^{2}$ follows directly from the fact that waves are compact and that each site topples once in a wave. Repeating the evaluation performed already twice, we find an exponent $\tau_{s}=11 / 8$ for the first wave, which is identical to the analytically derived exponent for the last wave [10], and different from the exponent $5 / 4$ for boundary avalanches in a sector of $360^{\circ}$ [3]. From the condition that waves are compact, it follows immediately that $\tau_{r}=7 / 4$ for the first wave, which means that a fraction proportional to $1 / L^{3 / 4}$ of all first waves reach the boundary. The result $\tau_{r}=7 / 4$ follows also directly from a scaling collapse of the radius distribution of dissipative first waves.

For the distribution of duration times $t$ of avalanches exactly the same analysis can be performed as for the number of topplings $s$. One finds again a violation of FSS, the typical duration time of an avalanche of radius $r$ being $\sim r^{z}$ with $z=4 / 3$, the upper cutoff of the duration time being larger than $r^{3 / 2}$, and the mean duration time of avalanches, averaged over all avalanche sizes, being $\bar{t} \sim L$.

The following table summarizes the results for the ex- 
ponents:

\begin{tabular}{r|c|c|c|c|c} 
& $\tau_{r}$ & $\tau_{t}$ & $\tau_{s}$ & $z$ & $D$ \\
\hline $1^{\text {st }}$ wave & $7 / 4$ & $8 / 5$ & $11 / 8$ & $5 / 4$ & 2 \\
avalanche & $3 / 2$ & $11 / 8$ & $11 / 9$ & $4 / 3$ & $9 / 4$
\end{tabular}

In summary, we have shown that the scaling behavior of the abelian sandpile model in two dimensions is tied to the condition that the fraction $1 / \sqrt{r}$ of all avalanches started at a distance $r$ from the boundary reach the boundary, rather than to a self-similarity of large and small avalanches. As a consequence, the scaling properties are seen most clearly from the dissipative avalanches, and a violation of FSS can occur.

The condition that a fraction proportional to $1 / \sqrt{L}$ of all avalanches reach the boundaries, together with the two conditions $\bar{s} \sim L^{2}$ and $\bar{t} \sim L$ seem so simple that they should be derivable from simple argument. In fact, $\bar{s} \sim L^{2}$ follows from the diffusive motion of sandgrains [2], and appears to be also valid in higher dimensions [11]. The first condition is equivalent to the statement that the probability of triggering a system spanning avalanche, $p$, is proportional to the density of surface sites, $\rho$, that topple during a system-spanning avalanche. The reason is that the product $p \rho L^{d-1}$ is the mean number of grains dissipated during an avalanche, which must be identical to 1 , resulting in $p \sim \rho \sim 1 / L^{(d-1) / 2}(d$ denotes the dimension of the system). A condition $p \sim \rho$ holds for example for percolation clusters in systems above the percolation threshold. It also holds at the percolation threshold, if there are only a finite number of spanning clusters in the system. In three dimensions, this condition would lead to $\tau_{r}=2$, which agrees with the simulation result given in [6]. If avalanches are compact, as suggested by computer simulations, the exponent $\tau_{a}$ characterizing the area distribution in three dimensions is then $\tau_{a}=4 / 3$, giving a mean avalanche area $\sim L^{2}$. Together with the condition $\bar{s}=L^{2}$, it follows that the mean number of topplings is of the same order as the mean area, leading to $\tau_{s}=4 / 3$ and to the conclusion that the number of waves of topplings remains finite in the thermodynamic limit, and that FSS is not violated in three dimensions. Finally, a compact avalanche of $\bar{s}=L^{2}$ sites has a radius $L^{2 / d}$. In two dimensions, this corresponds to the mean avalanche duration $\bar{t}$. If we assume the same in three dimensions, we have $\bar{t} \sim L^{2 / 3}$, and we find $\tau_{t}=8 / 5$, again in agreement with simulation results $\sqrt{6}$. In dimensions above the upper critical dimension 4 12, avalanches are no more compact, and the system may contain many spanning clusters, and the above arguments can therefore not be applied.

It thus seems that the scaling behavior of the abelian sandpile model follows from a few simple principles, a connection which still has to be explored in greater depth.

\section{ACKNOWLEDGMENTS}

This work was supported by EPSRC Grant No. GR/K79307.

[1] P. Bak, C. Tang and K. Wiesenfeld, Phys. Rev. Lett. 59, 381 (1987); Phys. Rev. A 38, 364 (1988).

[2] D. Dhar, Phys. Rev. Lett. 64, 1613 (1990).

[3] E.V. Ivashkevich, D.V. Ktitarev and V.B. Priezzhev, J. Phys. A: Math. Gen. 27, L585 (1994).

[4] S.S. Manna, J. Stat. Phys. 59, 509 (1990).

[5] A. Chessa, H.E. Stanley, A. Vespignani, and S. Zapperi, Phys. Rev. E 59, R12 (1999).

[6] S. Lübeck and K.D. Usadel, Phys. Rev. E 55, 4095 (1997).

[7] C. Tebaldi, M. De Menech, and A.L. Stella, condmat/9903270.

[8] M. De Menech, A.L. Stella, and C. Tebaldi, Phys. Rev. E 58, R2677 (1998);

[9] V.B. Priezzhev, D.V. Ktitarev, and E.V. Ivashkevich, Phys. Rev. Lett. 76, 2093 (1996).

[10] D. Dhar and S.S. Manna, Phys. Rev. E 49, 2684 (1994).

[11] P. Grassberger and S.S. Manna, J. Phys. France 51, 1077 (1990).

[12] V.B. Priezzhev, cond-mat/9904054. 